\title{
EDV-gestützte Fahrzeugdisposition und -abrechung im Baubereich zur Optimierung der Prozesskette
}

\author{
Prof. DR.-ING. DIPL.-WIRTSCH.-ING. W.A. GÜNTHNER, \\ DIPL.-ING. S. KESSLER, \\ DIPL.-ING. S. SANLADERER \\ LEHRSTUHL FÜR FÖRDERTECHNIK MATERIALFLUSS LOGISTIK, TU MÜNCHEN
}

\section{Zusammenfassung}

Die deutsche Bauindustrie sieht sich in der jüngeren Vergangenheit mit starkem Konkurrenzdruck von den neuen EU Mitgliedern konfrontiert, in dem sie sich nur durch technologischen Vorsprung behaupten kann, da einer reiner Preiskampf mit den Billiglohnländern mittelfristig durch die hohen Arbeitskosten in Deutschland nicht gewonnen werden kann. Der erforderliche technologische Vorsprung ist nicht nur auf die eigentlichen Bauprozesse beschränkt, sondern betrifft vor allen auch Prozesse wie die Baustellenlogistik, die mit der eigentlichen Wertschöpfung am Bauprojekt nichts zu tun haben. Durch den Einsatz von mobilen Datenerfassungsgeräten (Onboard Units) können die Prozesse zur Versorgung von Baustellen transparenter gestaltet und so ein automatisiertes Controlling zur Kostenssenkung eingeführt werden. Betriebsmittel können so effektiver eingesetzt werden, aber auch Lieferprozesse durch die höhere Planungssicherheit durch eine EDV Unterstützung schlanker gestaltet und besser mit dem Bauablauf synchronisiert werden. Im Rahmen eines AiF Forschungsprojektes wurde am Lehrstuhl für Fördertechnik Materialfluss Logistik (fml) der TU München in Zusammenarbeit mit Industriepartnern ein Flottentelematiksystem für die Baubranche unter Berücksichtigung der besonderen Randbedingungen entwickelt und in einem langfristigen Pilotversuch getestet

\begin{abstract}
The German construction industry is confronted with great pressure of competition from the new EU States since the last few years which can only be faced by a technological lead. A competition by price with low pay countries can in middle term not be won because of the high personal costs in Germany. The technological lead is not only necessary in the construction processes, but also in the processes not directly involved in the added value of a construction Building, eg. construction site logistics. Processes for the supply of the construction site get more transparent by the application of mobile computers (Onboard Units) and a automated controlling for cost reductions can easily be installed. Available equipment can be used with more efficiency, delivery processes can be designed more lean due to a higher quality in planning and get synchronised better with the construction development. In the scope of an Aif research project and in cooperation with industrial partners the institute for materials handling material flow and logistics (Fördertechnik Materialfluss Logistik - fml) of the Technical University of Munich, developed a fleet telematik system for the construction industry.
\end{abstract}

\section{Forschungsziel}

Das Ziel des Projektes liegt in der Optimierung der Transportkette (Transportminimierung, Erhöhung der Auslastung) am Bau, analog den industriellen Logistikoptimierungen der externen Transportkette (JIT, JIS). Durch die Bildung eines Informationsnetzes für die Logistik der Baustelle sollen die Transportprozesse transparenter werden, um bei Problemen oder unerwarteten Ablaufänderungen durch abweichende Baurandbedingungen frühzeitig steuernd in den Prozessablauf eingreifen zu können. Hierzu kann ein Transfer von Erkenntnissen der industriellen Logistik im Bereich der Ablaufplanung und des Logistikcontrollings, unter Berücksichtigung der speziellen Rahmenbedingungen, auf die Baubranche erfolgen. Weiterhin soll eine lückenlose, automatische und fehlerfreie Datenerfassung von transportbezogenen Daten (Zeiten, Mengen, Temperatur etc.) dargestellt werden, um das Qualitätsmanagement der Bauleistung zu vereinfachen. Die dezentral in den Fahrzeugen erfassten Daten sollen neben einer Qualitätsdokumentation auch für eine automatisierte Abrechnung sowohl der erbrachten Leistung als auch von lohnbezogenen Angaben für die Fahrer verwendet werden. Durch den Einsatz eines Telematiksystems in der Fahrzeugdisposition und -abrechnung sollen die variablen Kosten gesenkt und gleichzeitig die erbrachte Leistung und Qualität des gesamten Transportprozesses erhöht werden (siehe Abb. 1): 


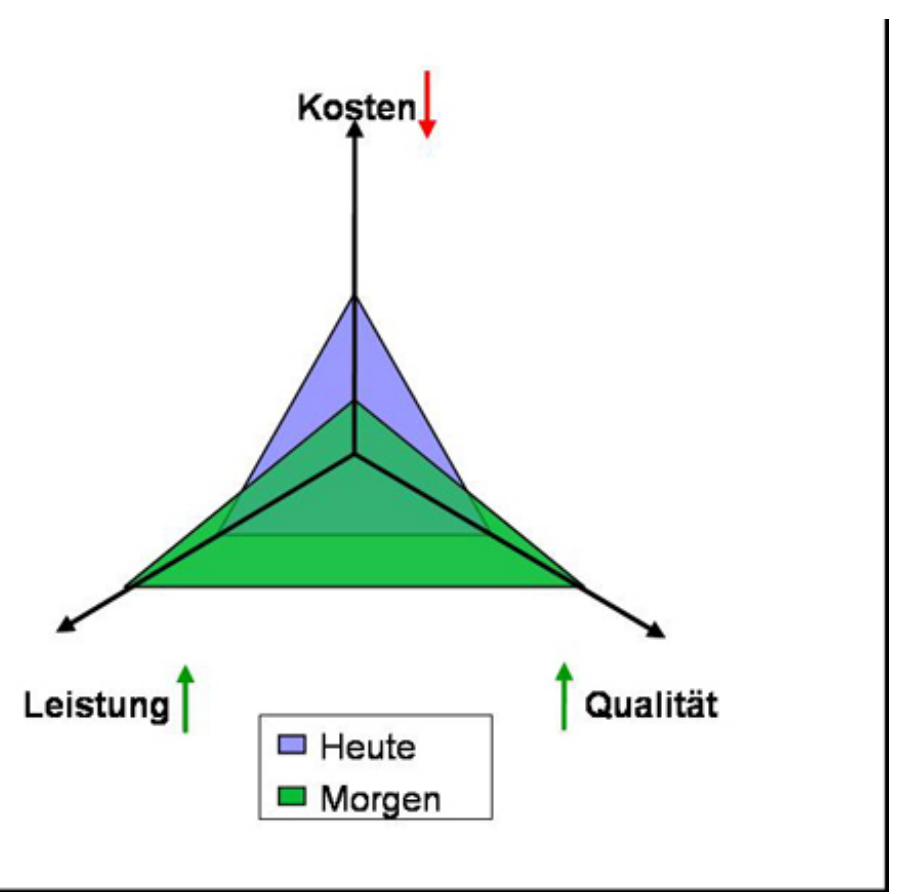

Abbildung 1: Globale Ziele der Transportlogistik am Bau

\section{Logistik im Baubetrieb}

Die Logistik hat in Industrie und Handel in den letzten Jahren sehr stark an Bedeutung gewonnen und stellt einen sehr wichtigen Wettbewerbsfaktor für die Unternehmen dar. Die Zeit- und Materialpuffer im Materialfluss der Produktion werden immer weiter optimiert um Kosten zu senken und die Durchlaufzeiten zu reduzieren. Diese Entwicklung zeigt sich in der Baubranche noch nicht in einer derartigen Ausprägung. Erste Ansätze für eine Logistiksteuerung am Bau beschränken sich in den meisten Fällen auf den baustelleninternen Materialfluss. Baustellenübergreifende Koordination findet in der Regel nicht statt, da jede Großbaustelle solitär versucht ihre internen Abläufe zu optimieren, ohne dabei globale logistische Zusammenhänge zwischen den jeweiligen Baustellen zu berücksichtigen. Um den Einsatz einer Baustellenlogistik-Steuerung auch unter wirtschaftlichen Gesichtspunkten zu rechtfertigen, sollte die Baustelle aber eine gewisse Größe aufweisen oder unter besonderem Zeitdruck oder kritischen Rahmenbedingungen abzuwickeln sein. Bei wachsender Größe der Baustellen wird es für den Bauleiter durch die Vielzahl an Einflussparametern ungleich schwieriger alle Leistungen optimal zu planen und die notwendigen Transporte und Materialien zum richtigen Zeitpunkt anzufordern [Eichler98]. Der Aufwand einer EDV-gestützten Baustellenlogistikplanung wird sich durch den hohen Nutzen, der mit derartigen Systemen verbunden ist, relativ schnell rechnen. Auf Kleinbaustellen dagegen wird der Aufwand zur Einführung eines Logistikkonzeptes sich erst sehr spät oder unter Umständen gar nicht amortisieren, da keine großen Einsparungspotentiale gegeben sind. Bei kleinen bis mittleren Baustellen ist in der Regel die Koordination durch den Bauleiter oder Polier ausreichend, wenn dieser die nötige Erfahrung hat.

Ein wichtiger Teil der Logistik am Bau ist die Planung und Abwicklung der Transporte zur Ver- und Entsorgung der Baustelle, die in diesem Forschungsvorhaben optimiert werden soll. In der Abbildung 2 sind schematisch die Lieferketten einiger wichtiger Quellen und Senken entlang des gesamten Bauprozesses dargestellt: 


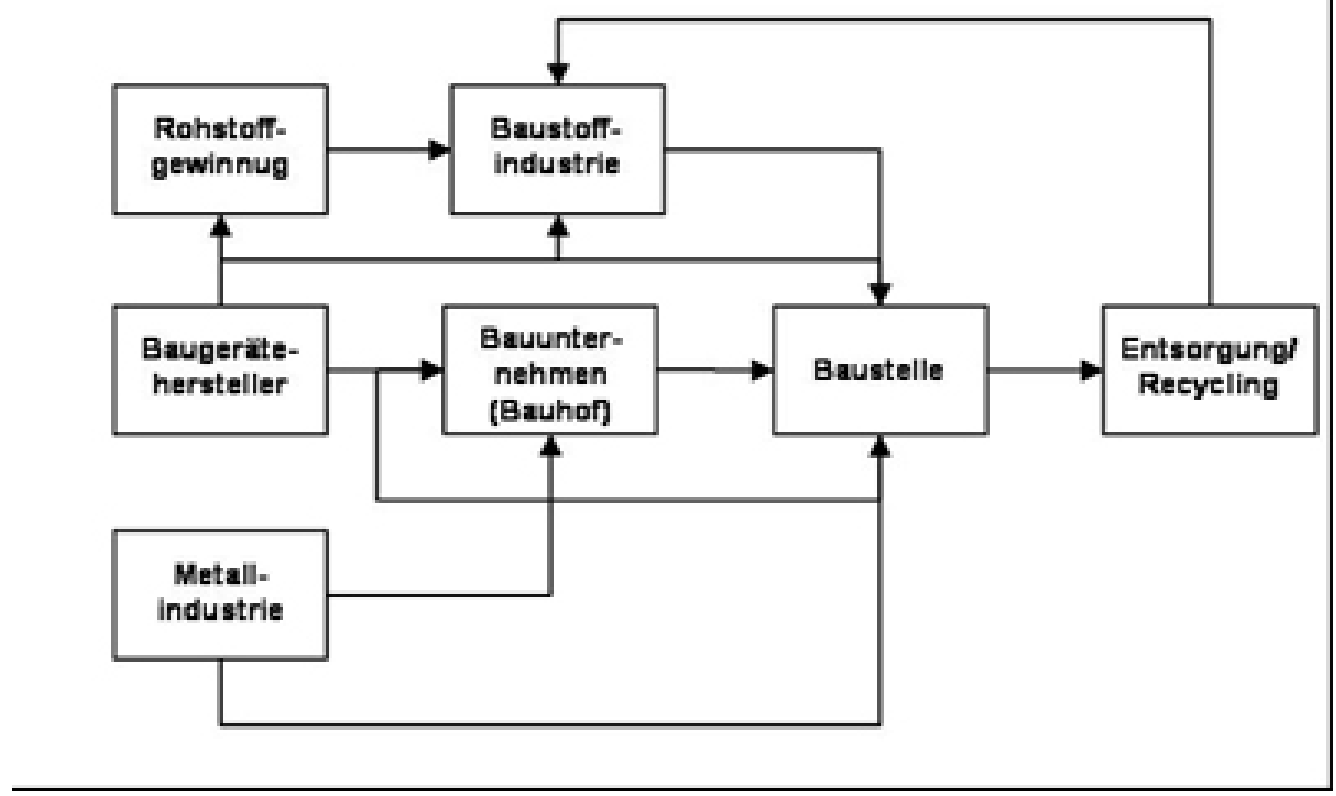

Abbildung 2: Lieferketten der Bauindustrie

Transportgüter im Baubereich bilden ein breites Spektrum der Transportanforderungen. Im Stückgutbereich müssen Kleinteile, wie Gitterboxen und Kleingeräte, bis hin zu großen Baumaschinen mit Sonderzulassung nach StVZO kostenoptimal auf die Baustelle gebracht werden. Bei den Stückgütern fallen sehr große Transportmengen an, die es optimal zu koordinieren gilt, um transportbedingte Verzögerungen auf den Baustellen zu verhindern. Abbildung 3 zeigt einen kurzen Überblick der beiden Transportgruppen Stück- und Schüttgüter:

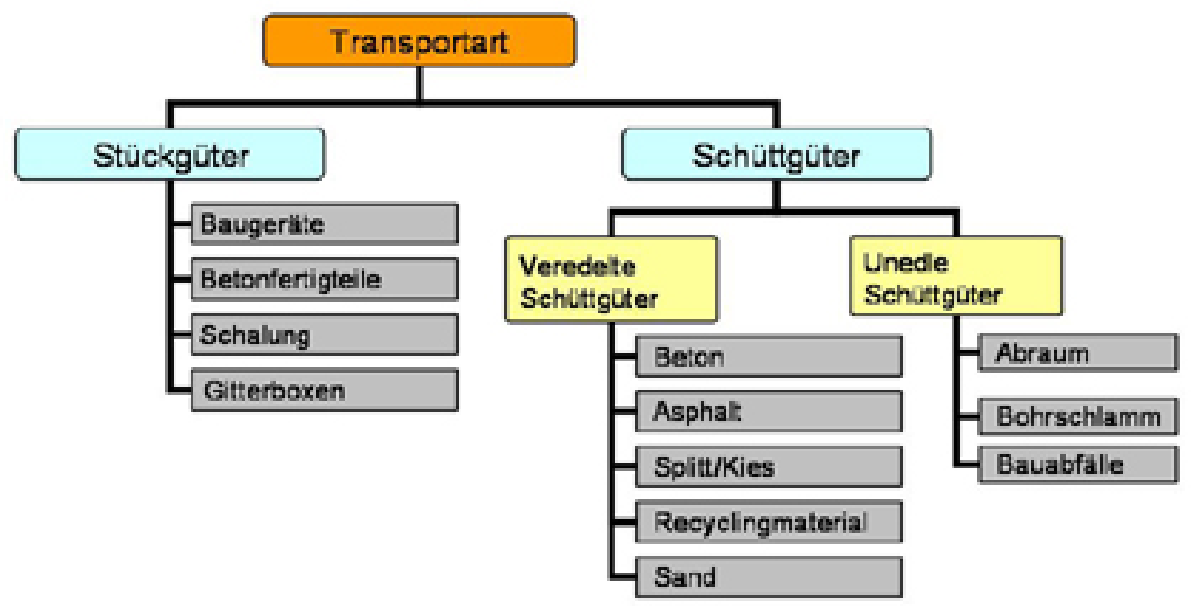

Abbildung 3: Übersicht der Transportgüter einer Bauunternehmung 


\section{Rahmenbedingungen für das Transportwesen im Baubereich}

Seit dem 01. Mai 2006 ist durch die Verordnung EG 2135/98 für Neufahrzeuge mit einer Gesamtmasse von über 3,5 Tonnen die Einführung des digitalen Tachographen (DT) obligatorisch. Der digitale Tachograph ist die Basis für eine effektivere Kontrolle der Einhaltung der EU-Vorschriften über die Lenk- und Ruhezeiten des Fahrpersonals.

Ziel ist eine Erhöhung der Verkehrssicherheit, eine Vereinfachung und Vereinheitlichung im Umgang mit dem Kontrollgerät sowohl für den Fahrer, als auch für Kontrollorgane und Unternehmen [Padrtka06].

Für Baufirmen mit einem eigenen Fuhrpark ergeben sich dadurch wichtige Änderungen in der Einsatzplanung sowohl der Kraftfahrer als auch die Fahrzeuge. Der Disponent muss die Lenk- und Ruhezeiten auf Grund der umfangreichen Kontrollmöglichkeiten seitens der Behörden deutlich exakter einhalten als dies in der Vergangenheit der Fall war. Die Auslastung der LKW sollte aber dennoch möglichst hoch sein, um die Flotte effizient zu nutzen und weiterhin wettbewerbsfähig zu bleiben.

Wegen dieser geänderten Rahmenbedingungen müssen auch für die Baubranche neue, flexible Arbeitszeitmodelle entwickelt werden, die aber für die Administration/Disposition einen höheren Planungsaufwand bedeuten. Eine rein manuelle Planung, wie sie aktuell in vielen klein- und mittelständischen Bauunternehmen betrieben wird, wird somit noch weiter erschwert, da der Disponent bei der Transportplanung zusätzliche Parameter berücksichtigen muss. Eine gute Disposition wird wir noch stärker abhängig von einer detaillierten Informationsbasis bezüglich der tatsächlichen Fahrzeiten der LKW/Fahrer sowie der Tourenplanung oder der LKW-Position. Für diese Aufgabenstellung kann mit Hilfe eines Fahrzeugtelematiksystems durch eine an die Baubranche angepasste Technik eine gute Unterstützung der Dispositionsarbeit erzielt werden.

Neben rechtlichen Rahmenbedingungen gilt es bei der Konzeption eines Logistiksystems für das Transportwesen am Bau auch die branchenspezifischen Besonderheiten zu berücksichtigen. Konzepte der konventionellen Logistik sind nicht eins zu eins auf den Bausektor übertragbar, da die Baubranche einer Vielzahl von Randbedingungen unterliegt, die sowohl auf die bautechnischen Prozesse als auch auf die Baulogistik Einfluss nehmen. Nachfolgend sind einige dieser Faktoren stichpunktartig dargestellt:

- Jedes Bauwerk bzw. Bauvorhaben ist ein individuelles Projekt mit ganz speziellen Anforderungen und Restriktionen. Die Planung muss an jede Baustelle adaptiert werden. Erfahrungen aus früheren Abwicklungen können meist nicht eins zu eins übertragen werden, sondern lediglich als Orientierung für das Projektmanagement dienen.

- Die Fertigung der Gewerke ist nur begrenzt von der Baustelle zu entkoppeln, etwa durch den Einsatz von Fertigteilen. Daraus resultiert eine starke Abhängigkeit der Materialflussplanung von bautechnisch bedingten Wartezeiten zur Trocknung und Aushärtung der Baustoffe, die nicht beeinflusst werden können.

- Eine Lagerhaltung ist auf den Baustellen aufgrund des begrenzten Platzbedarfs nur in beschränktem Umfang und auch nur für ein begrenztes Sortiment möglich. Viele Güter werden direkt nach der Anlieferung verbaut und sollten auch möglichst JIT angeliefert werden.

- Im Schüttgutbereich ist bei Fahrzeugen mit nur einer Ladefläche kein Splitting der Fracht möglich, und somit sind auch Systeme zur Tourenoptimierung nur bedingt einsetzbar. Nur ca. $10 \%$ des gesamten Transportbedarfs einer Bauunternehmung ist Teilladungsverkehr mit verschiedenen Entladestellen, während die restlichen 90\% Ganzladungsverkehr mit direkter Belieferung einer Baustelle darstellen.

- $\quad$ Bei der Transportplanung sind sehr unterschiedliche Anforderungen des zu transportierenden Gutes zu berücksichtigen. Die Bandbreite der Güter ist sehr weit gefächert.

- Eine Disposition der Fahrzeuge auf den Baustellen analog der Steuerung von LKW des Speditionsbereichs über einzelne Fahraufträge ist pauschal nicht möglich, da im Bereich des Erdbaus die Fahrstrecken meist nur sehr kurz sind und die Lieferorte im Voraus oft nicht planbar sind. Vorhandene Planungssysteme sind für diese Anforderungen zu unflexibel und bieten auch nicht die notwendigen Schnittstellen um zusätzliche Informationen für die Bauabwicklung gewinnen zu können.

- Starke Abhängigkeit vom Wetter, demzufolge auch sehr große Belastungsspitzen der Fahrer während der Sommermonate. Für ein automatisiertes Planungssystem sind diese diskontinuierlichen Leistungsbedarfe nur schwer zu verarbeiten, da sie im Normalfall von gleichförmigen Anforderungsprofilen ausgehen.

- Stückgüter sind meist Spezialanfertigungen, daher ist eine Standardbeladungsplanung über Optimierungssoftware zur Ladeflächenauslastung nur bedingt möglich. Bei vorgespannten Betonfertigteilen beispielsweise bestehen Belastungsrestriktionen, da nur bestimmte Flächen aufliegen dürfen, um die Teile nicht zu beschädigen. 


\section{Telematik zur Optimierung der Transportabwicklung}

Telematiksysteme im Speditionsgewerbe werden üblicherweise mit einer Dispositionssoftware im Hintergrund betrieben, können aber auch als „Stand-alone“ Lösung in den Fahrzeugen eingesetzt werden. Ohne eine Dispositionssoftware, die mit hohen zusätzlichen Investitions- und Lizenzkosten verbunden ist, kann eine günstige Lösung erzielt werden, welche sich durch Reduzierung der variablen Kosten relativ schnell amortisieren. Als Beispiel hierfür kann die „Fleetmanager“ Bordeinheit von VDO Kienzle zur Erfassung der Arbeitszeit, Kilometer und Spritverbrauch, sowie der gefahrenen Geschwindigkeit genannt werden.

Durch Reduzierungen des Spritverbrauch und der Überstundenanzahl um ca. 20\% wird der Return on Invest (ROI) sehr schnell erreicht. Der Einsatz bei einer mittelständischen Bauunternehmung amortisiert sich durch die geringen Investitionskosten bereist nach knapp vier Monaten [Götz00].

Mit einer zusätzlichen Dispositionssoftwarelösung erhöhen sich zwar die Investitionskosten stark, dafür kann ein derartiges System zur unternehmensübergreifenden Optimierung der Logistikprozesse eingesetzt werden. Somit ergeben sich nicht nur für die Transportabteilung des Unternehmens sondern für die gesamte Bauunternehmung zusätzliche Vorteile aufgrund der schnellen Datenübermittlung und lückenlosen automatischen Dokumentation einer Vielzahl von Vorgängen.

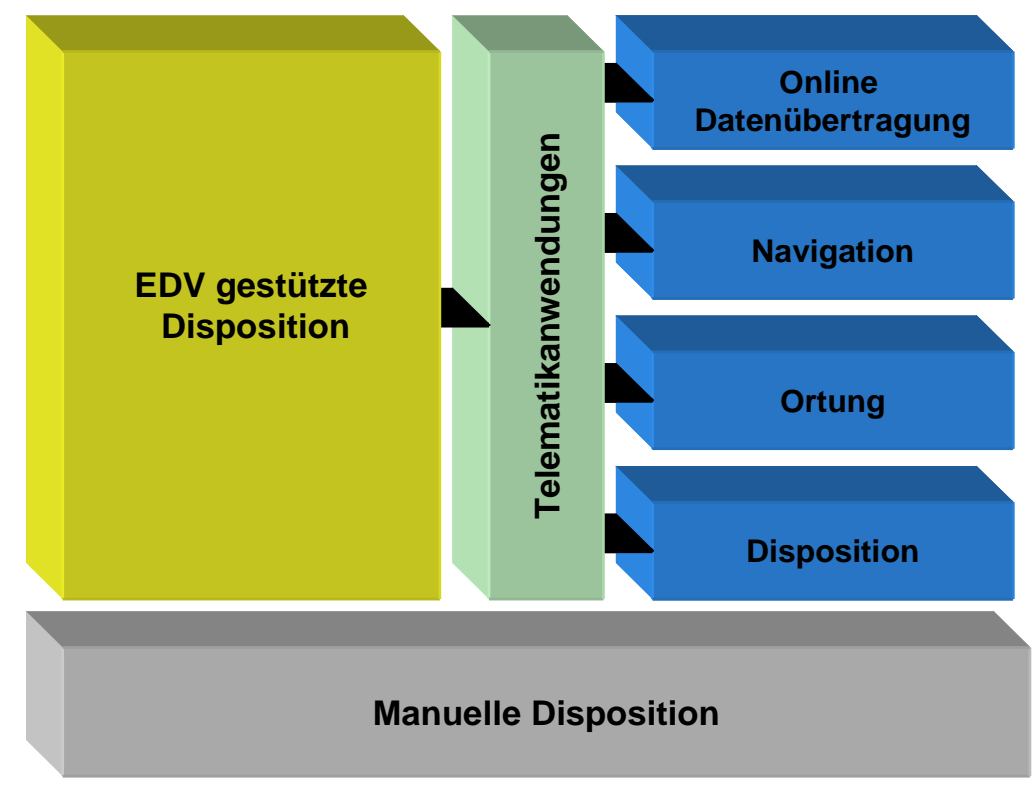

Abbildung 4: Klassifizierung von Dispositionssystemen

Im Bereich der Dispositionssoftware werden von den am Markt befindlichen Lösungen vorwiegend Funktionen für die klassische Speditionsabwicklung im Stückguttransport und Teilladungsverkehr abgebildet. Der Schwerpunkt liegt vorwiegend in der Mehrdepottourenplanung und -optimierung zwischen verschiedenen Auslieferstandorten. Dieses Tool, welches im Bereich der Speditionen und Paketdienste die Kernfunktion darstellt, ist aber für den Bausektor weniger relevant, da die meisten Transporte zu den Baustellen (ca. 95\%) im Ganzladungsverkehr durchgeführt werden [Schmalz00][Andres02]. Speziell für den Bau gibt es derzeit allerdings noch keine geeignete Lösung. Der Strukturwandel des Transportsektors zwingt die Unternehmen, ihre Kostenreduzierungspotentiale zu erkennen und auch zu nutzen, um weiterhin am Markt erfolgreich arbeiten zu können. Dadurch ändern sich auch die Anforderungen an die Disponenten.

\section{Transportabläufe bei der Baustellenversorgung}

Die administrativen Prozesse für eine Transportlogistik am Bau sind aktuell stark geprägt von manuellen Tätigkeiten zur Datenerfassung und -kontrolle. Der Material- und Informationsfluss zwischen Baustelle, Disposition und den Lieferwerken ist in folgender Abbildung erläutert: 


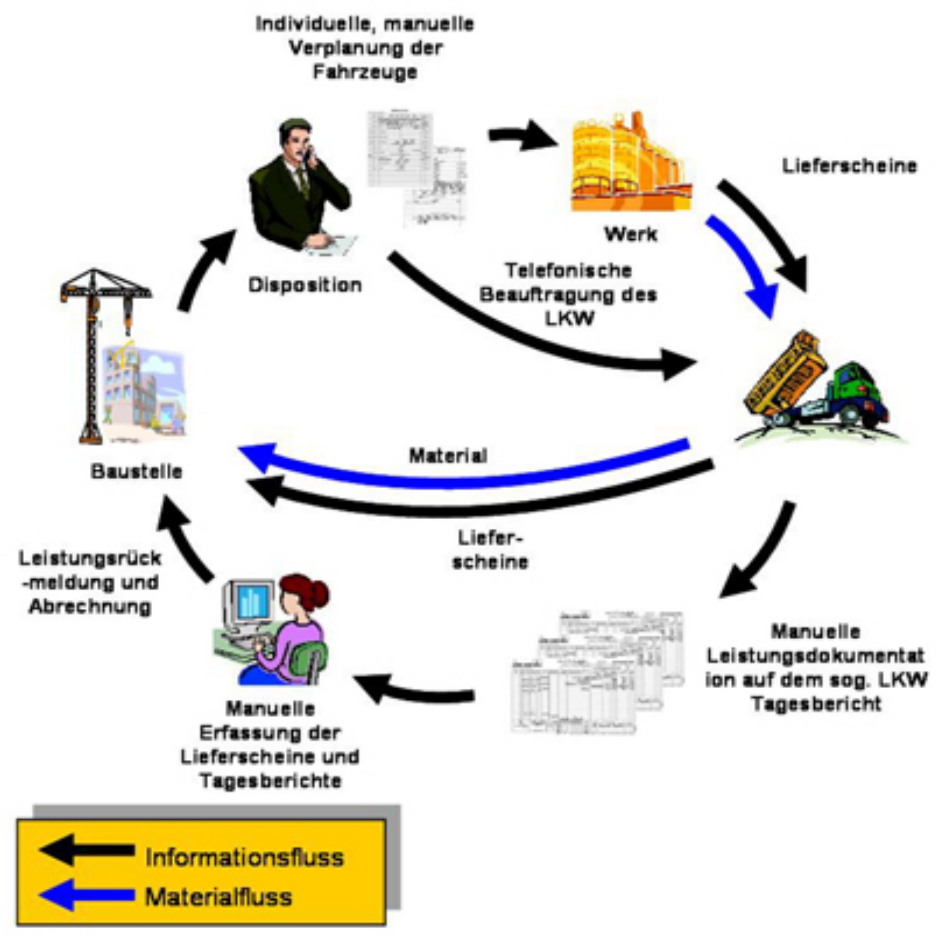

Abbildung 5: Ablauf eines Baustellentransports - Material- und Informationsfluss

Ziel des Telematikeinsatzes ist die Optimierung der gesamten Prozesskette von den Produktionswerken bis hin zur Belieferung der Baustellen mit Schüttgütern, wie Asphalt-Mischgut, Transportbeton sowie Betonfertigteilen. Neben der reinen Fahrzeugdisposition sind die beiden weiteren Hauptziele des Transportlogistik-Projektes die durchgängige Integration der Lohn- und Leistungsabrechnung sowie die Erfassung von Qualitäts- und Leistungskennwerten für die Kunden. Durch die Eingliederung in bestehende Back-Office Systeme können am Bordrechner erfasste Daten automatisiert verarbeitet und manuelle Arbeitsschritte zur Abrechnung so weit als möglich vermieden werden. Somit reduziert sich der Zeitbedarf und Arbeitsaufwand zur gesamten Auftragsabwicklung erheblich.

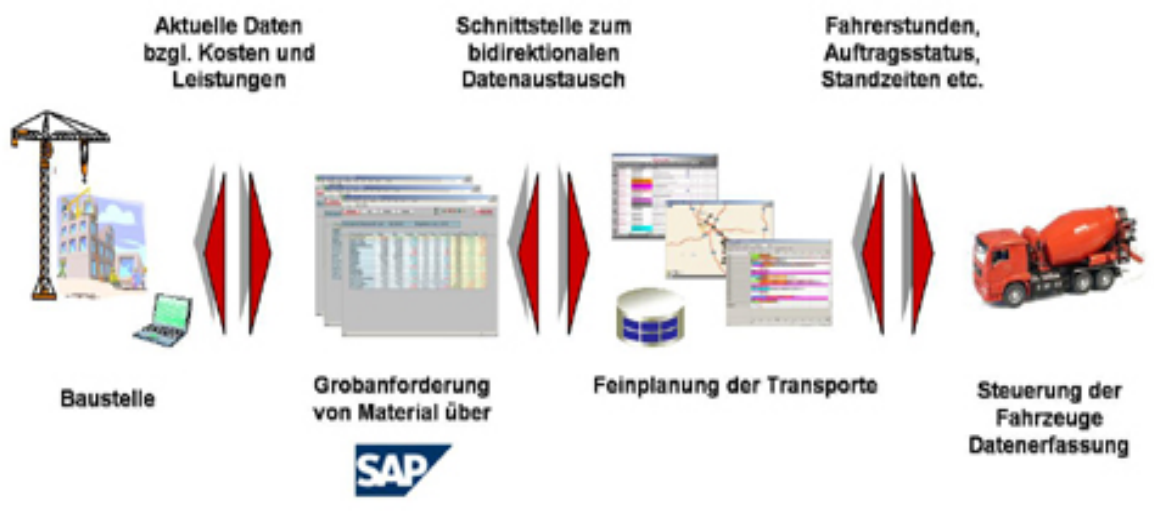

Abbildung 6: EDV-gestützter Dispositions- und Abrechnungsprozess

Zeitnahe statistische und abrechnungsrelevante Auswertungen sowohl für die Kunden, als auch für interne Stellen können mit geringem Aufwand generiert und den Nutzern zur Verfügung gestellt werden. So können einerseits die Transportkosten durch den optimierten Einsatz der Fahrzeuge gesenkt und andererseits eine lückenlose digitale Qualitätsdokumentation gewährleistet werden. 
Zusätzlich können aus den protokollierten Daten der Fahrzeuge Leistungskennwerte generiert und dem Kunden für statistische Zwecke zur Verfügung gestellt werden. Weiterhin soll die Planungssicherheit und Transparenz der externen sowie internen Transporte auf der Baustelle verbessert werden. Dies betrifft einerseits die Zusammenarbeit mit den Baustellen und Werken im Vorfeld der Planung, um die Transporte optimal abwickeln zu können, andererseits auch die Kontrolle der Fahrzeuge während des Tages durch eine Onlineübertragung von Statusdaten, die dem Disponenten jederzeit Auskunft über die Tätigkeit seiner LKW liefern. Der Fuhrpark wandelt sich somit zu einem integrierten Dienstleister, der durch die Bereitstellung von qualitativ hochwertigen Daten hinsichtlich Leistung und Aufwand einen Mehrwert sowohl für die Baustelle, als auch für die Lieferwerke generiert, da alle Beteiligten besser in den Material- und Informationsfluss integriert werden.

Im Zuge des beschriebenen Forschungsprojektes wurde in Zusammenarbeit mit den Partnern aus der Industrie ein Demonstrator entwickelt, der in einem langfristigen Praxiseinsatz getestet wurde und für weitere Einsatzzwecke weiterentwickelt werden soll.

\section{Hardware}

Die Hardware, die in den LKWs verbaut ist, besteht aus einem Bordrechner, der an die Fahrzeugelektronik zur Erfassung der gefahrenen Kilometer und der Kippvorgänge angeschlossen wird, und einem Touchscreen-Display zur Bedienung (siehe Abb. 4) durch den Fahrer. Der Rechner ist universell einsetzbar und kann im Gegensatz zu den herstellerbezogenen Insellösungen für Fahrzeugtelematiksysteme in LKW verschiedener Hersteller problemlos eingebaut werden. Für gemischte Flotten kann somit ein einheitliches Gerät eingesetzt werden, bei dem alle Fahrer unabhängig vom aktuellen Fahrzeug immer mit demselben System arbeiten können.

Bei der Auswahl der Geräte wurde besonders auf eine bedienerfreundliche sowie robuste Ausführung geachtet, da die Geräte durch den Einsatz speziell innerhalb der Baustelle harten Umgebungsbedingungen, vor allem durch die Vibrationen und Verschmutzung, ausgesetzt sind.

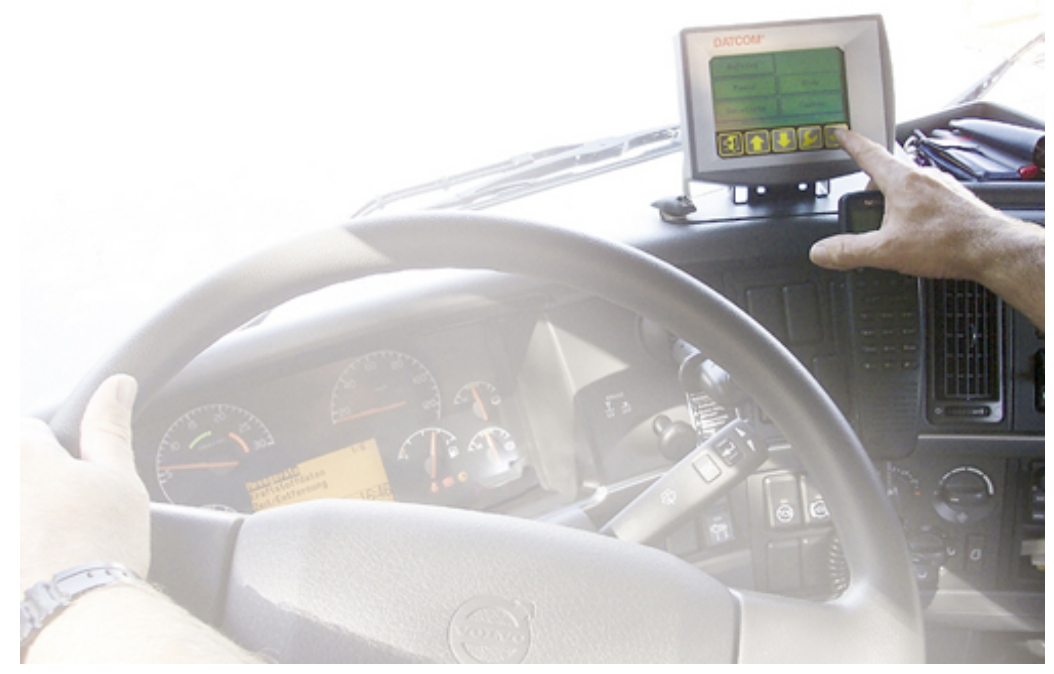

Abbildung 7: Einbaubeispiel Bordrechner [Foto Max Bögl]

Die Kommunikation mit den Bordrechnern erfolgt über GPRS (General Packet Radio Service), da eine Echtzeitübertragung der Daten erforderlich ist, damit der Disponent während des Tages schnell auf Änderungen oder Probleme im Transportablauf reagieren kann. Der Disponent hat somit die Möglichkeit, „online“ die Arbeit der Fahrzeuge zu überwachen und, wenn nötig, sofort in den laufenden Prozess einzugreifen. Für diesen Einsatzfall bietet GPRS im Vergleich zu einer Datenübertragung per sms deutliche Kostenvorteile, da beim datenbasierten GPRS nicht die Anzahl der Verbindungen, sondern lediglich die übertragenen Datenmengen Kosten verursachen. Mit Hilfe eines GPS-Empfängers wird permanent die Fahrzeugposition bestimmt, so dass bei jeder Statusmeldung der aktuelle Standort an das zentrale System übermittelt werden kann.

Zur Gewährleistung der Übertragungssicherheit verfügen die Bordrechner über einen Sicherheits-Puffer, der bei fehlender Funkverbindung alle Status- und Auftragsmeldungen zwischenspeichert und alle bis dahin aufgelaufenen Meldungen an die zentrale Datenbank sendet, sobald wieder eine Netzverbindung besteht. 


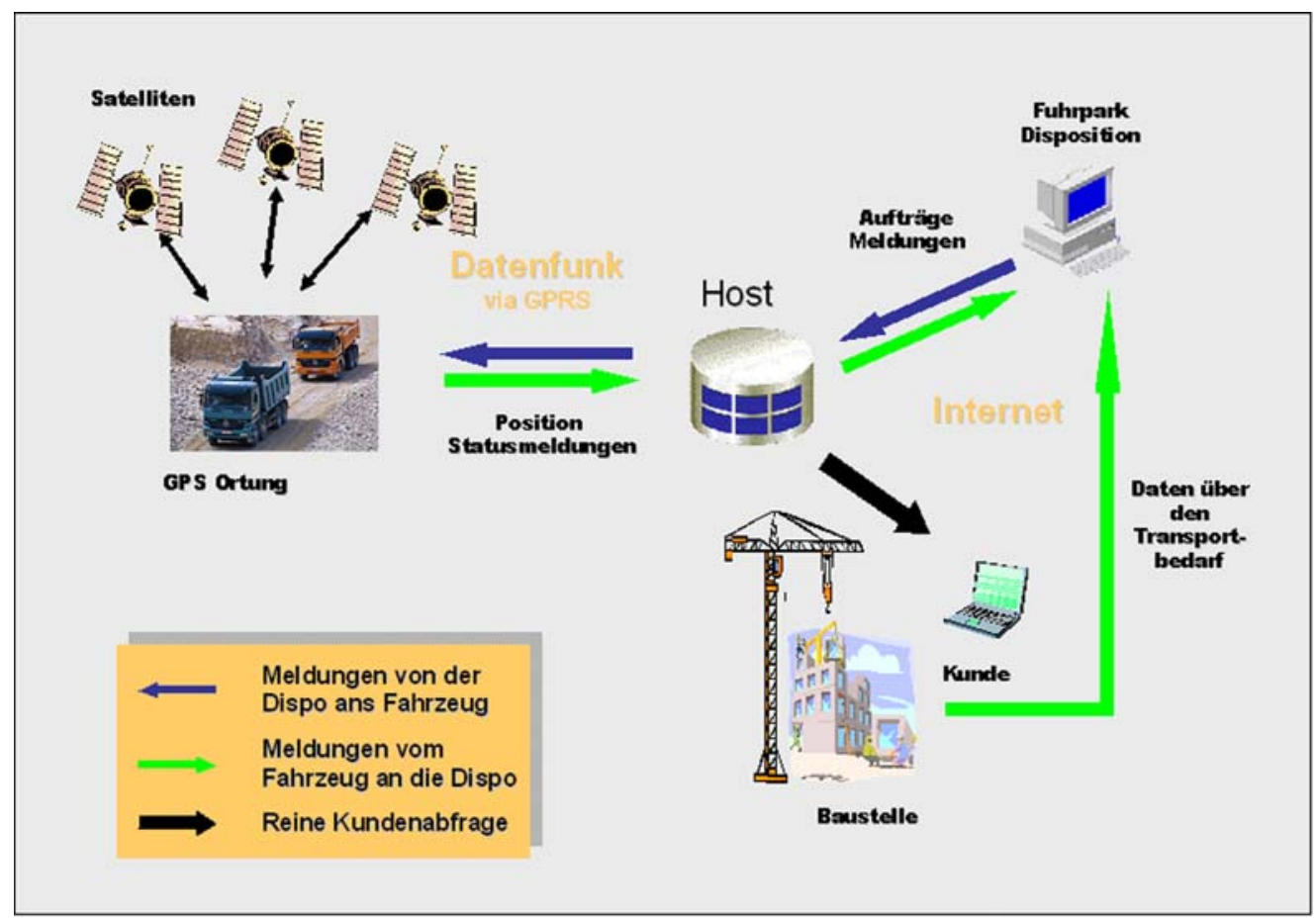

Abbildung 8: Funktionsskizze Telematiksystem

Die gesamte Kommunikation mit den Fahrzeugen wird über einen zentralen Internetserver (Host) abgewickelt, der die Daten auch für weitere Softwareapplikationen zur Verfügung stellt (siehe Abb. 8).

\section{Software}

Zur Disposition der Fahrzeuge stehen dem Nutzer zwei Softwaremodule für die Auftrags- und Fahrzeugverwaltung zur Verfügung, die ihn bei der Planung der Transportaufträge unterstützen:

- „Contour Web“ Kernfunktionen des Systems (Auftrags- und Stammdatenverwaltung, Kommunikation etc.)

- „Shipping Manager“ Planungsunterstützung durch graphische Anzeige der Aufträge auf die LKW auf einer Zeitachse (siehe Abb. 9)

Die eingesetzte Dispositionssoftware ist internetbasiert und bietet dem Disponenten somit die Möglichkeit seine Fahrzeuge von jedem Arbeitsplatz mit Internetzugang aus zu verwalten, ohne dass an diesem PC eine umfangreiche Installation des Programms erforderlich ist. Lediglich das Modul „Shipping“ muss noch separat einrichtet werden. Im Hauptmodul "Contour Web" werden alle Stammdaten bezüglich Fahrzeugen, Anhängern, Baustellen, Fahrern etc., die zur Auftragserstellung und -bearbeitung notwendig sind, verwaltet. Zur Gewährleistung der Datenaktualität werden neue Lieferorte bzw. Kunden über eine Schnittstelle aus SAP dynamisch aktualisiert.

Der Disponent generiert seine Transportaufträge mit Angabe der Abhol- und Lieferadresse sowie den zugehörigen Zeiten und produktspezifischen Daten über dieses Modul. Die Zuordnung eines Auftrages auf ein Fahrzeug kann er entweder selbst durch Auswahl eines bestimmten LKW übernehmen oder alternativ automatisiert durch das Programm durchführen lassen. Über Optimierungsalgorithmen wird dann aus allen bestehenden Aufträgen und verfügbaren LKW die beste Fahrzeugauswahl für die Transportaufträge berechnet und als Vorschlag im zweiten Softwaremodul „Shipping Manager“ angezeigt. Als Kriterien für die automatische Fahrzeugauswahl werden Parameter, wie beispielsweise die Verfügbarkeit der Fahrzeuge, die Entfernung zum Abholort oder maximale Nutzlast der verfügbaren Fahrzeuge verwendet.

Der Disponent kann nun entweder den Systemvorschlag akzeptieren oder zusätzliche manuelle Änderungen der Auftragsverteilung vornehmen. Solange die Aufträge noch nicht versendet wurden, können durch einfache Drag\&Drop-Funktionalitäten die Aufträge zwischen verfügbaren Fahrzeugen einfach ausgetauscht werden. Transportaufträge, die bereits versendet wurden, müssen zunächst zurückgerufen werden, um Änderungen vorzunehmen. Die Anzeige im „Shipping Manager“ ist dynamisch und wird laufend durch die rückgemeldeten Statusdaten der Fahrzeuge aktualisiert. 


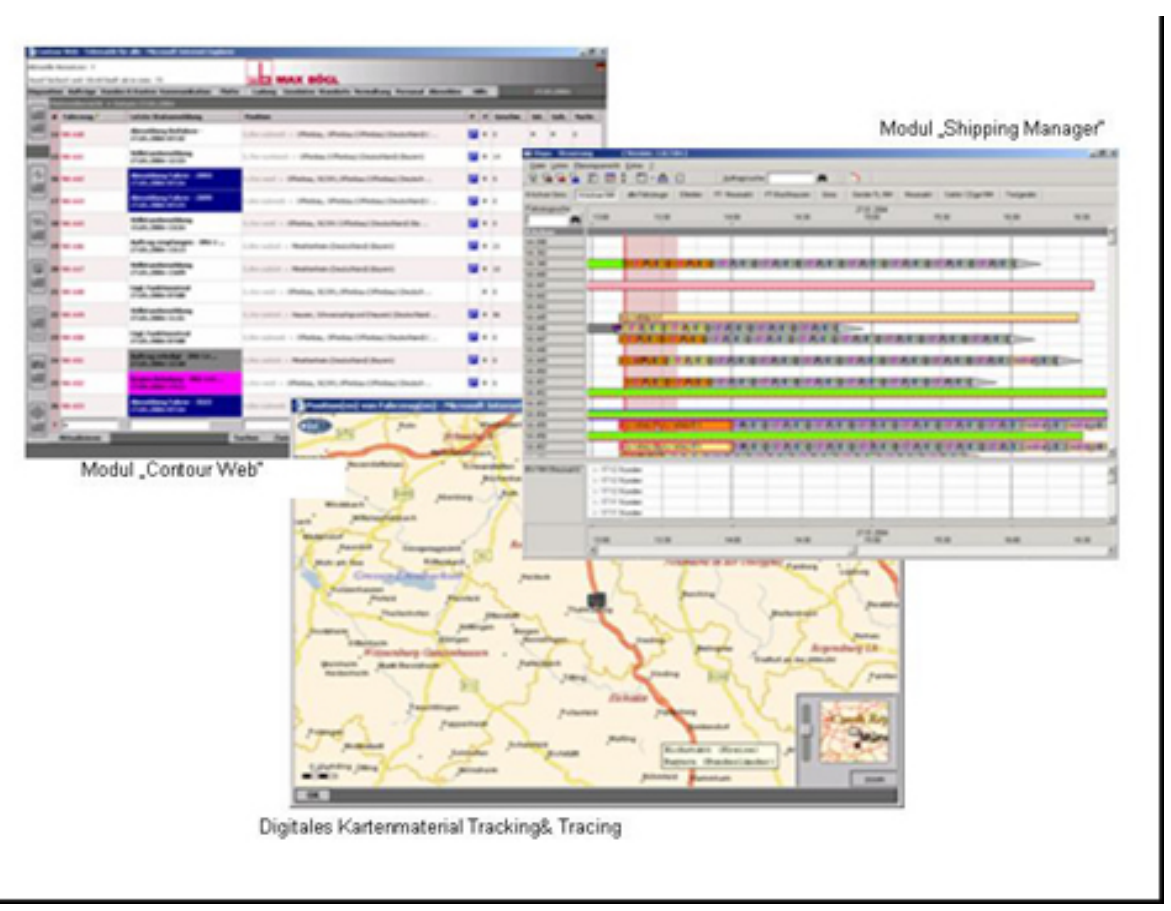

Abbildung 9: Dispositionssoftware

Die Bordrechner in den Transportfahrzeugen erfassen alle fahrer- und auftragsbezogenen Meldungen, die für eine spätere automatisierte Abrechnung notwendig sind. Hierzu zählen beispielsweise Arbeitsbeginn und -ende, Pausenzeiten, Nebentätigkeiten wie Fahrzeugpflege etc. Alle Informationen eines Transportauftrags, wie Beginn des Beladevorganges Zeitpunkt der Ankunft an der Entladestelle oder Lieferscheinnummer, werden direkt digital verarbeitet und müssen nicht zusätzlich über eine manuelle Schnittstelle im EDV System verbucht werden. Informationen stehen sofort zur Verfügung und können nahezu in Echtzeit ausgewertet werden. Neben auftragsbezogenen Daten können mit dem Bordrechner auch fahrzeugspezifische Daten, wie gefahrene Kilometer, Geschwindigkeit etc., automatisch erfasst und dokumentiert werden. Zu jeder Aktion, die im Fahrzeug generiert wird, überträgt der Bordrechner die aktuelle Uhrzeit und die GPS-Koordinaten, so dass ein durchgängiges Tracking\&Tracing (siehe Abb. 9) der Fahrzeuge möglich wird.

\section{Einsparspotenziale durch das Telematiksystem}

Durch die mobile Datenerfassung im LKW können alle Informationen eines Transportauftrags sowie die zugehörigen lohnrelevanten Daten, wie Zeiten, zuschlagsberechtigte Tätigkeiten etc. der Fahrer direkt digital erfasst und an die jeweiligen Systeme zur Verrechnung übertragen werden. Eine zusätzliche manuelle Verbuchung wie bisher, die mit hohem Zeit- und Kostenaufwand verbunden ist, kann somit entfallen. Das Telematiksystem ermöglicht weiterhin ein objektives Controlling der Fahrzeuge, da alle Prozessbeteiligten (Fahrer, Disponenten) in einem einheitlichen System arbeiten. Die gewonnen Daten sind gut miteinander vergleichbar, da subjektive Einflüsse bei der Datenerfassung weitgehend ausgeschlossen werden. Strategische Entscheidungen, wie der Einsatz von 4-Achsern oder Dumpern im Baustelleneinsatz, können somit durch praxisrelevante Leistungsdaten aus vergangenen Projekten gestützt werden.

Standzeiten der Fahrzeuge werden durch die zeitnahe Rückmeldung der Statusdaten sehr schnell erkannt und der Disponent kann korrigierend eingreifen, indem er den Grund für Standzeiten (Zu geringe Entladekapazitäten, zu viele Fahrzeuge in einem Umlauf, defektes Einbaugerät auf der Baustelle etc.) - falls möglich - beseitigt. Durch eine verbesserte Planung und höhere Transparenz des Transportprozesses kann bereits im Vorfeld das Risiko von Stillstands- bzw. Wartezeiten auf der Baustelle minimiert werden. Unnötige Nebenzeiten der Fahrer, wie beispielsweise die zusätzliche manuelle Leistungsdokumentation in einem LKW-Tagesbericht, können durch die direkte EDV-gestützte Datenerfassung an den Bordrechnern entfallen. Somit wird der produktive Zeitanteil eines Fahrzeugs wesentlich erhöht und die Effektivität des gesamten Fuhrparks steigt deutlich.

Neben den direkten Einsparungsmöglichkeiten in der Abrechnung und Disposition der LKW, die über die Zeiteinsparung bzw. Erhöhung der Auslastung gut monetär quantifizierbar sind, ergeben sich durch den Bordrechnereinsatz in der Zusammenarbeit mit der Baustelle noch zusätzlichen Mehrwert, der nur schwer finanziell zu bewerten ist. Hierzu zählen vor allem die bessere Informationsbereitstellung für die Baustelle und die höhere Transparenz der Transportvorgänge. Die Abläufe innerhalb der Baustellen können durch die genaue Erfassung aller Fahrzeuge, die mit Bordrechnern ausgestattet sind, wesentlich besser überwacht und geregelt 
werden. Für die Baustelle reduziert sich somit der Aufwand für die Erstellung einer Leistungsdokumentation, da auf die Daten der Fahrzeuge zurückgegriffen werden kann.

Zur Gesamtauswertung müssen diese dann lediglich noch mit Daten, die nicht bzw. noch nicht automatisch erfasst werden, kombiniert und in der gewünschten Form dargestellt werden.

\section{Fazit}

Durch steigenden Wettbewerbs- und Kostendruck sowie geänderte rechtliche Rahmenbedingungen bieten Telematiksysteme auch für den Baustelleneinsatz einen sehr hohen Nutzen, wenn sie konsequent in einem durchgängigen Konzept eingesetzt werden, das auf die individuellen Bedürfnisse der Anwender zugeschnitten ist. Durch die automatisierte Auftragsverwaltung können weit reichende Einsparpotenziale aufgezeigt werden, die sich nicht nur auf die Transportabteilungen im Unternehmen beschränken. Neben der Fahrzeugdisposition ergeben sich durch den Bordrechnereinsatz für Baufirmen noch eine Vielzahl weiterer Nutzungsmöglichkeiten, wie beispielsweise die Verfolgung von Massenströmen auf den Baustellen oder die Aufzeichnung von qualitätsrelevanten Daten, wie Transportdauer oder -temperatur, die vom Benutzer je nach Anforderung automatisch dokumentiert werden können. Damit können behördlich geforderte Qualitätsmerkmale problemlos dokumentiert und später bei Bedarf nachgewiesen werden.

Neben dem Einsatz von Onboard Units im LKW bieten sich noch zahlreiche weitere Anwendungsgebiete für eine mobile Datenerfassung und -bearbeitung am Bau, beispielsweise in den Baumaschinen oder zur Leistungsund Stundenerfassung des Bau-Personals. Baufirmen können durch den Einsatz von Telematiksystemen ihre Kosten effektiver kontrollieren und reduzieren und sich durch eine Verbesserung des gesamten Prozessablaufs (synchronisierte Abläufe, Qualitätsdokumentation etc.) bereits heute sehr gut für die Aufgaben von morgen rüsten.

\section{Literatur}

[Padrtka06]

[Eichler98]

[Andres02]

[Schmalz00]

[Götz00]
Padrtka, Ralf: Infoline zur Einführung des digitalen Tachographen, Verkehrsrundschau http://www.digitaler-tachograph.com

Eichler, Peter: „Baustellenlogistik Berlin Potsdamer Platz“ in: Internationales Verkehrswesen, Ausgabe 11/98

Andres, Marco: „Telematiksysteme für die e-Logistik - Anwendungsweise, Lösungen, Marktüberblick“, FTK Forschungsinstitut für Telekommunikation, Dortmund, 2002“

Schmalz, Andreas: „Zentrale versus dezentrale Transportplanung - Eine vergleichende Analyse für Multi-Depot Tourenplanungsprobleme“, Veröffentlichung Justus Liebig Universität Gießen, 2000 Götz, Hans-Jürgen: „Big Brother am Bau“, IN: Firmen Auto (2000) 8 